\title{
Advanced application of a sublayer fence probe in highly instationary turbomachinery flows: observations on prestall instabilities
}

\author{
Mario Eck ${ }^{1} \cdot$ Roland Rückert ${ }^{1} \cdot$ Dieter Peitsch ${ }^{1}$
}

Received: 28 August 2018 / Revised: 21 January 2019 / Accepted: 22 January 2019 / Published online: 26 February 2019

(c) The Author(s) 2019

\begin{abstract}
The present paper introduces a novel approach for measuring near wall flow velocities by utilizing a sublayer surface fence probe. Hereby, a difference in static pressure builds up over a microscopic obstacle within the viscous sublayer. An analytical model of the angular dependent pressure difference is employed to derive information about the flow direction. Furthermore, a computational preston tube approach has been used to calibrate the surface fence probe with regard to a flow velocity to be assigned to half of the fence height. Through the use of a sophisticated analyzing algorithm the flow direction and its velocity can be determined as a function of time. As a proof of concept measurements were conducted within the radial gap of an annular compressor rig yielding both mean and time resolved near wall flow fields. Former are in very good compliance with oil flow visualizations proving the methods accuracy. The experimental results give unprecedented insights into an unsteady flow phenomenon that arises when an axial compressor is operating close to its stalling limit. The presented technique allows for investigating turbomachinery areas which formerly were observable only by computational means.
\end{abstract}

\section{Graphical Abstract}

Mario Eck

mario.eck@tu-berlin.de

$\triangle$ Roland Rückert

roland.rueckert@tu-berlin.de

1 Institut für Luft- und Raumfahrt, Marchstraße 12-14, 10587 Berlin, Germany

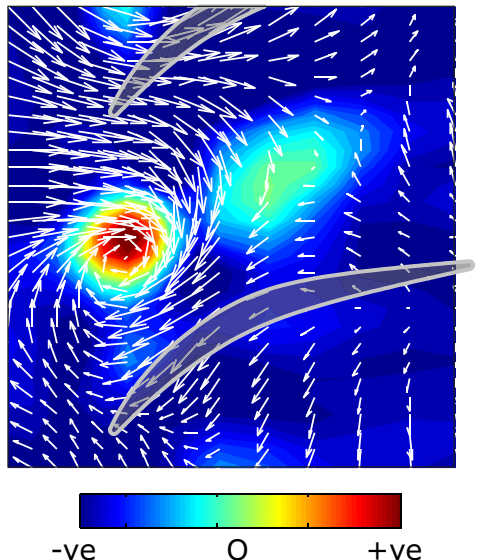

\section{List of symbols}

Abbreviations

CPM Computational preston tube method

CSC Compressor stator cascade

FP Focus point

LE Leading edge

MAE Mean absolute error

MEMS Micro electro mechanical systems

PS Pressure side 


$\begin{array}{ll}\text { PSD } & \text { Power spectral density } \\ \text { RI } & \text { Rotating instability } \\ \text { RMS } & \text { Root mean square } \\ \text { SFP } & \text { Surface fence probe } \\ \text { SP } & \text { Saddle point } \\ \text { SS } & \text { Suction side } \\ \text { TE } & \text { Trailing edge } \\ \text { VIGV } & \text { Variable inlet guide vane }\end{array}$

\section{Greek letters}

$\beta \quad$ Flow angle $\left({ }^{\circ}\right)$

$\Delta \quad$ Difference (-)

$v \quad$ Kinematic viscosity (-)

$\Omega \quad$ Vorticity tensor (1/s)

$\phi \quad$ Circumferential angle $\left({ }^{\circ}\right)$

$\rho \quad$ Density $\left(\mathrm{kg} / \mathrm{m}^{3}\right)$

$\tau_{\mathrm{W}} \quad$ Wall firction $\left(\mathrm{kg} / \mathrm{m}^{3}\right)$

$\omega \quad$ Probe rotation angle $\left({ }^{\circ}\right)$

\section{Latin letters}

$\tilde{t} \quad$ Sequence time (-)

$B, C \quad$ Regression curve coefficients (-)

$c \quad$ Chrod length (m)

$d \quad$ Diameter of preston tube (m)

$f \quad$ Frequency $(\mathrm{Hz})$

$h \quad$ Height of the suface fence (m)

$i \quad$ Incidence angle $\left({ }^{\circ}\right)$

$K \quad$ Displacement factor (-)

Ma Mach number (-)

$p \quad$ Static pressure (Pa)

$Q \quad$ Vortex criterion (-)

$q \quad$ Dynamic pressure $(\mathrm{Pa})$

$R_{c} \quad$ Casing diameter (m)

$R_{h} \quad$ Hub diameter (m)

$S \quad$ Strain tensor (-)

$t \quad$ Time (s)

$U \quad$ Inflow velocity to the compressor stator $(\mathrm{m} / \mathrm{s})$

$u^{+} \quad$ Non-dimensional velocity (-)

$u_{F} \quad$ Surface fence velocity $(\mathrm{m} / \mathrm{s})$

$u_{P} \quad$ Preston tube velocity $(\mathrm{m} / \mathrm{s})$

$u_{\tau} \quad$ Shear stress velocity $(\mathrm{m} / \mathrm{s})$

$v \quad$ Axial velocity component $(\mathrm{m} / \mathrm{s})$

$y^{+} \quad$ Non-dimensional wall distance (-)

$y_{\text {eff }} \quad$ Effective wall distance (m)

\section{Introduction}

A common method to measure fluctuating shear stresses by means of magnitude and direction is the employment of so called surface or sublayer fences. This implicit measuring technique is able to provide directional information of the adjacent mean wall shear stress and its absolute value. The measurement principle was first introduced by Patel (1965) and later adopted on the basis of micro-electro-mechanical systems (MEMS) (Von Papen et al. 2002; Schober et al. 2004). The present work utilizes this method in an annular compressor rig demonstrating its capabilities in general turbomachinery applications. As of now, it has been challenging to examine areas in-between outer casing walls and the adjacent blades from a fluid mechanics point of view. The present work proposes an approach to overcome those difficulties.

Through the use of a sophisticated trigger method which is found within the examined and highly instationary flow field the surface fence probe allows for measuring time resolved secondary flow structures. Furthermore, the data reduction method first introduced by Dambach and Hodson (1999) and later adapted by Weichert and Day (2013) is being improved. The provided analyzing scheme represents a time efficient and accurate method which can also be applied to varying shear stress measuring techniques such as MEMS and surface hotwire-gauges in general.

\section{Measurement principle}

The basic principle of surface or sublayer fences respectively is based on a differential pressure $\Delta p$ which builds up between the up- and downstream location of a microscopic obstacle when fluid is crossing the same. Not only does $\Delta p$ depend on the flows direction but also on its absolute velocity. The former relationship is going to be discussed in the first part of this paper whereas the latter will be discussed subsequently. The sublayer fence probe (SFP) probe used for the present work consists of a fence-like impediment, 0.15 $\mathrm{mm}$ in height and is fitted with two fast response pressure transducers, namely Meggitt $8507 \mathrm{C}$-1, to either side of the fence, see Fig. 1. To ensure high sensitivity in $\Delta p$ fluctuations, the transducers are being located in close proximity to the taps and the fence. Specifications of the sensors are summarized in Table 1.

The probe has been manufactured by means of a CNC milling machine. Therefore, the minimum step resolution set the
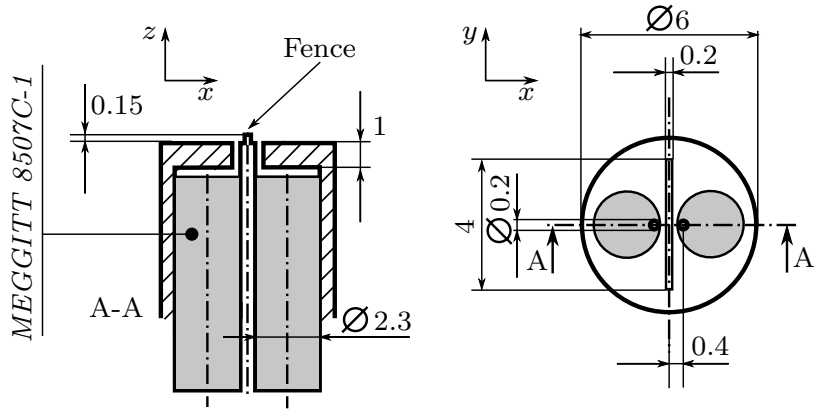

Fig. 1 Sublayer fence probe and its dimensions in $\mathrm{mm}$ 
Table 1 Specifications of pressure transducers (Meggitt 8507C-1) embedded in the SFP

Diameter

Pressure range

Full scale output (FSO)

Non-repeatability

Resonance frequency

fence height to $0.15 \mathrm{~mm}$. As a rule of thumb, the length of the fence has to be at least 20 times the taps diameter (Nitsche and Brunn 2006) which has been fulfilled in this configuration. The outer diameter of the probe is $6 \mathrm{~mm}$ due to the parallel arrangement of the pressure transducers inside the probes body.

A simplified linear analysis of unsteady pressure measuring systems was carried out to analytically estimate the frequency response of the line-cavity system inside the SFP. For this purpose the Poiseuille relation for the pressure drop associated with laminar flow in a pipe () matching the line-cavity geometry has been solved. The relation is expressed by the following differential equation:

$l \frac{\mathrm{d}^{2} U}{\mathrm{~d} t^{2}}+k_{1} \frac{\mathrm{d} U}{\mathrm{~d} t}+\frac{S}{\rho C} U=0$.

Here, $k_{1}=32 l v / d^{2}$ is a system-constant describing the pressure taps geometries, $S$ is the cross sectional area of the cavity and $C=V /\left(\kappa P_{0}\right)$ the fluidic capacitance of the cavity. Once the solution of the differential equation $U(t)$ is known, the time-resolved pressure $P_{3}$ at the end of the transducer cavity can be obtained according to:

$P_{3}(t)=1 / C \int_{0}^{t} U(t) \cdot S \cdot \mathrm{d} t$.

The frequency response of a step-wise increase in pressure is shown in Fig. 2. The natural frequency corresponds to the reciprocal of $\Delta t$ and is $f_{0}=3390 \mathrm{~Hz}$.

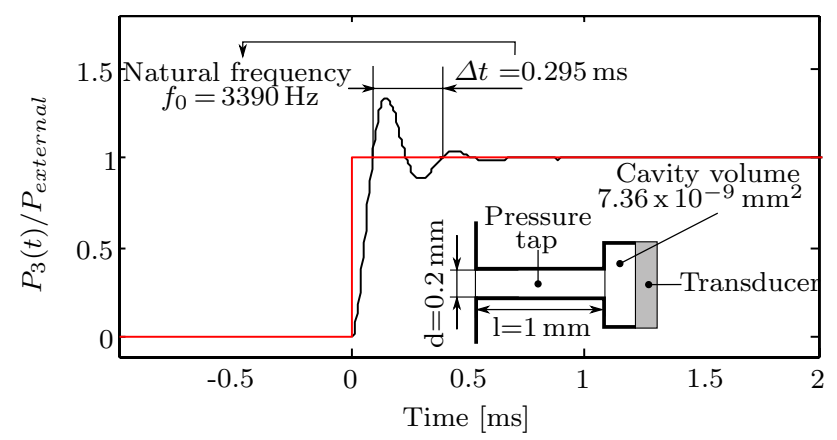

Fig. 2 Analytical pressure response of the line-cavity system schematically depicted in the subplot
For verification reasons a model giving a rough estimate for the frequency response of a Helmholtz-Resonator was used additionally. The underlying equation,

$f_{0}=\frac{a}{2 \Pi} \sqrt{\frac{\Pi d^{2} / 4}{V(l+\Pi d / 4)}}$,

yields a natural frequency of $f_{0}=3315 \mathrm{~Hz}$ which is in good compliance with the frequency derived from Fig. 2. The frequency which needs to be resolved in the later presented flow analysis is $f=300 \mathrm{~Hz}$ which is about $9 \%$ of the natural frequency of the line-cavity system. Thus, there is no need to apply an amplification or dampening function to the measured pressure fluctuations due to resonance effects inside the connecting volume.

Rotating the probe in a fully turbulent boundary layer without a pressure gradient yields the characteristic angular dependent distribution of $\Delta p=p_{1}-p_{2}$ shown in Fig. 3 . Here, the rotational step width is held constant at $\Delta \omega=5^{\circ}$.

In its normalized form, $\Delta p / \Delta p_{\max }=f(\omega)$ is independent of the main flow velocity. The maximum differential pressure corresponds to a fence position perpendicular with respect to the flow direction. Thus, to obtain the exact incoming flow angle $\beta$ one would have to adjust the probe's yaw angle $\omega$ by incremental steps until the maximum value of $\Delta p$ has been found. This time-consuming procedure is not feasible. Furthermore, a time resolved phase averaged observation of local flow phenomena, as will be discussed later, would not be possible. A solution to this problem is hereby presented which enables to approximate $\Delta p_{\max }$ utilizing trigonometric principles.

The angular dependent slope of the differential pressure can sufficiently be described by the classical cosine function as it has been done in former studies (Weichert and Day 2013). A more accurate approximation however can be established

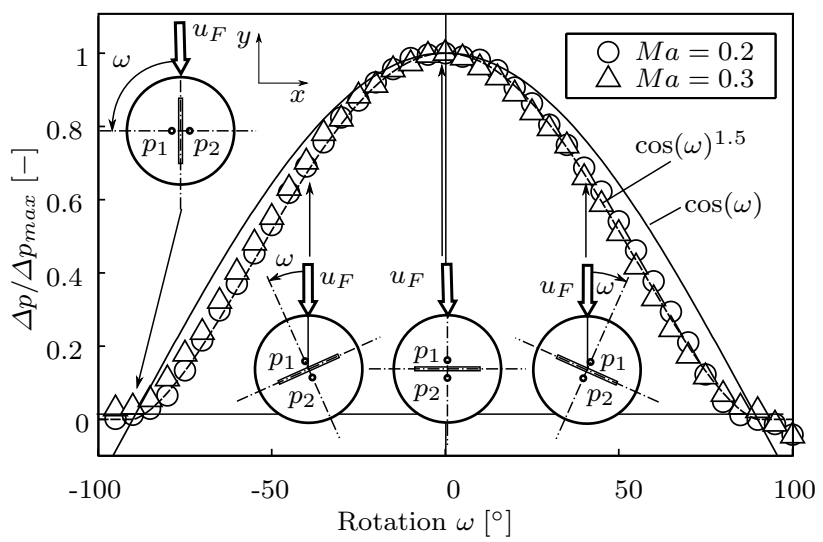

Fig. 3 Angular dependent distribution of the differential pressure at different Mach numbers 
through the introduction of a $\cos ^{1.5}$ distribution, as proposed by Nitsche et al. (1984). This way, the characteristic flattened out areas at $\omega= \pm 90^{\circ}$ are being appropriately accounted for. Vagt and Fernholtz (1973) adopted another approach given by the semi-empirical relation according to:

$\left.\frac{\Delta p}{\Delta p_{\max }}\right|_{\text {Fernh }}=\exp \left[-\left(1.025 \frac{\Pi}{180} \alpha\right)^{2}\right]$.

The various mean square errors (MSE) representing the deviation between the measured quantities $\Delta p / \Delta p_{\max }$ and the different approaches are summarized in Table 2 .

As the $\cos ^{1.5}$-function shows the smallest overall MSE, it is used throughout the following derivations.

Given any flow angle $\beta$ and rotating the SFP by the angle $\omega$ yields a phase-shifted $\cos ^{1.5}$-distribution as depicted in Fig. 4 .

The corresponding function is shown in Eq. 5.

$\Delta p=\Delta p_{\max } \cdot o s^{1.5}(\beta-\omega)$.

The two main parameters of interest are the incoming flow angle $\beta$ and $\Delta p_{\max }$ as it provides information about the local absolute velocity $u_{F}$ which needs to be calibrated based on a reference method (shown in Sect. 3).

After some rearrangement and through the use of basic trigonometry, expression 5 changes to:

$$
\begin{aligned}
\frac{(\sqrt[3]{\Delta p})^{2}}{\cos (\omega)}= & \left(\sqrt[3]{\Delta p_{\max }}\right)^{2} \sin (\beta) \tan (\omega) \\
& +\left(\sqrt[3]{\Delta p_{\max }}\right)^{2} \cos (\beta) .
\end{aligned}
$$

Thus resembling a linear equation of the form $A=B X+C$ where the individual coefficients are defined as followed:

$$
\begin{aligned}
& \boldsymbol{A}=\frac{(\sqrt[3]{\Delta p})^{2}}{\cos (\omega)}, \\
& \boldsymbol{B}=\left(\sqrt[3]{\Delta p_{\max }}\right)^{2} \cdot \sin (\beta), \\
& \boldsymbol{C}=\left(\sqrt[3]{\Delta p_{\max }}\right)^{2} \cdot \cos (\beta), \\
& \boldsymbol{X}=\tan (\omega) .
\end{aligned}
$$

Table 2 Mean square errors from the flow angle characteristic

\begin{tabular}{ll}
\hline Approach & MSE \\
\hline Cosine function & $7.2 \times 10^{-3}$ \\
Cosine $^{-1.5}$ function & $0.216 \times 10^{-3}$ \\
Fernholtz & $3.7 \times 10^{-3}$ \\
\hline
\end{tabular}

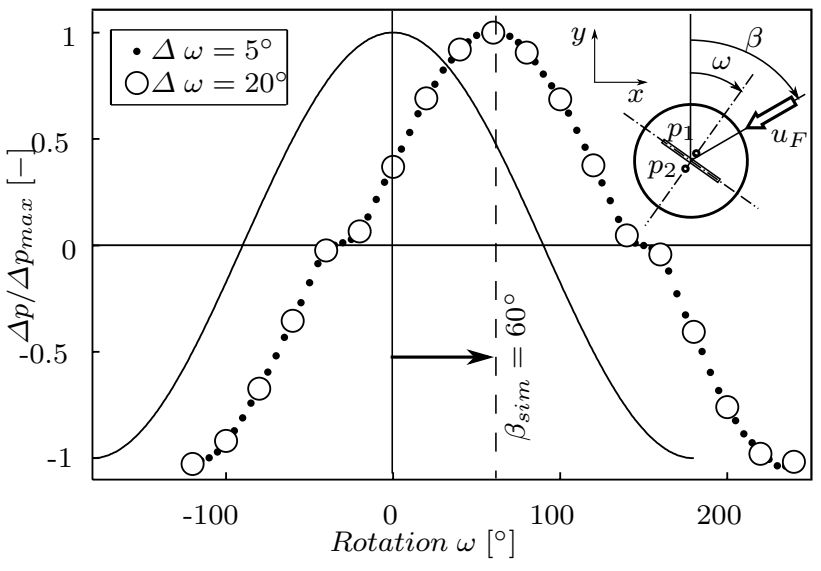

Fig. 4 Simulation of various inflow angles by shifting the experimentally obtained pressure distribution along the abscissa

Figure 5 illustrates how the foregone steps essentially result in a coordinate transformation, where the $\{\Delta p, \omega\}$-tuplets end up in a straight line, proving the underlying approximation function to be accurate. The coefficients $A$ and $X$ have been exemplary computed for two different $\Delta \omega$.

Any curve fitting tool can now be applied to derive the slope $B$ and the point of interception $C$. A recombination of those coefficients ultimately leads to the wanted physical properties:

$\beta=\arctan \left(\frac{B}{C}\right)$,

$\Delta p_{\max }=\left(\sqrt[4]{\frac{B \cdot C}{\sin (\beta) \cos (\beta)}}\right)^{3}$

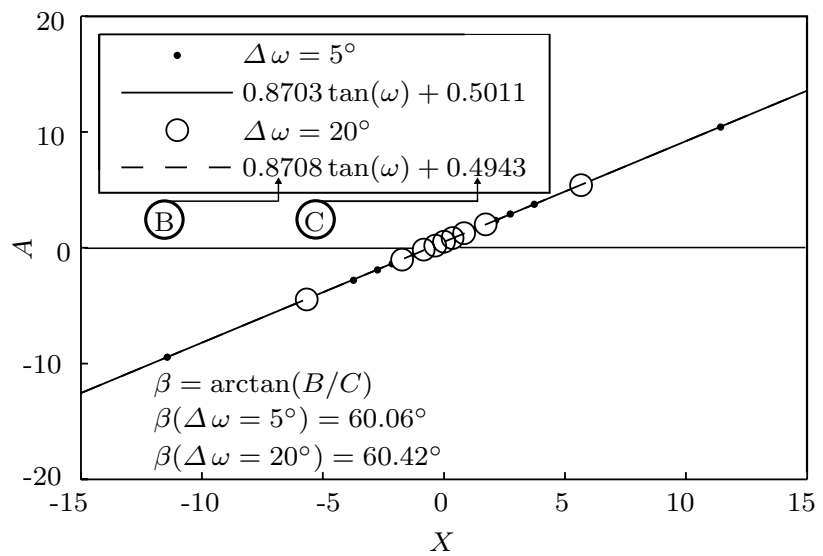

Fig. 5 Linearized $\Delta p(\omega)$ distribution at an incoming flow angle of $\beta=60^{\circ}$ 
The obvious advantage here is that the point associated with the maximum differential pressure and thus the flow direction can be calculated without the need to accurately pinpoint it. A finite number of yaw angle positions $\omega$ (at least four points) leads to an exact solution. To demonstrate the accuracy of this method, an error analysis is conducted. The algorithm is being fed with the experimentally obtained angular distribution recorded with a $5^{\circ}$ step width (cp. Fig. 3). As shown in Fig. 4 the data has been shifted to simulate different flow directions $\beta_{\text {sim }}$. Additionally, a variation of the angular resolution is simulated by only taking every 2 nd, 3 rd, 4 th, ... $\omega$ step into account. The absolute error shown here is derived by subtracting the preset flow angle $\beta_{\text {sim }}$ and the algorithm output $\beta$.

MAE $=\frac{\sum_{i=0}^{n}\left|\beta_{\text {sim }}-\beta\right|}{n}=\frac{\sum_{i=0}^{n}\left|e_{i}\right|}{n}$.

The newly implemented approximation shows superior behavior compared to the classical cosine approach over the whole range of flow angles, see Fig. 6. Also, the increase in step width to $\Delta \omega=20^{\circ}$ only marginally reduces the absolute accuracy compared to the smallest step width. A full sensor rotation is then being represented by 18 positions around its vertical axis $z$. Hence, this setup is used for the subsequent area measurements.

\section{Probe calibration}

It was shown that in order to identify the flow direction no reference-based calibration method is necessary. It is rather based on a mathematical relationship between the distribution

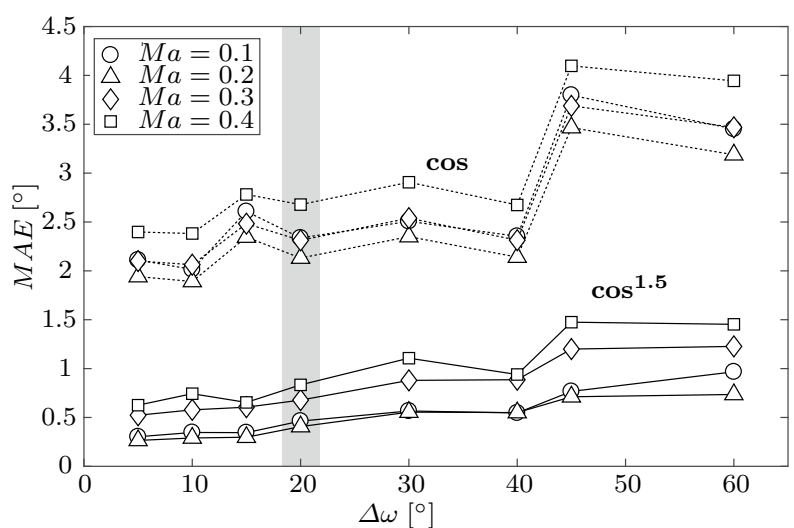

Fig. 6 Comparison of the mean absolute error of both approximation methods for different step widths and Mach numbers. For each Mach number the experimentally obtained angular distributions have been shifted in a step width of $\Delta \beta_{\text {sim }}=5^{\circ}$ leading to 72 processed $\beta$ that were used to calculate the error. The step width of $20^{\circ}$ marked in grey was adopted in the later experiments of the differential pressure and the flow direction. Up to this point, no absolute values of $\Delta p$ where looked at in detail. Commonly, the sublayer fence is calibrated for wall shear stresses using an appropriate reference measurement method. However, the present application has a different objective, namely the visualization of flow structures so that the pressure difference $\Delta p$ is set in relation to the velocity $u_{F}$ at half of the fence height. For this purpose, a reference measurement principle based calibration must also be performed.

Here, a microscopic 3D-printed preston tube was used to determine the near wall velocity to later calculate a corresponding velocity closer to the wall at half the height of the fence $h / 2$. The preston tupe method was used mainly motivated by its simplicity. In contrast to hot wire anemometry the preston tube does not require an additional reference based calibration that might introduce further errors to the very calibration procedure itself.

The computational preston tube method in its basic form, which the formerly described procedure is based upon, was first introduced and verified by Nitsche et al. (1985). It basically assigns local wall shear stresses to a measured dynamic pressure. It is based upon the similarity laws of the wall boundary layer where nondimensionality is achieved by introducing a shear stress velocity $u_{\tau}$ such that:

$u^{+}=\frac{u}{u_{\tau}}$,

$y^{+}=\frac{u_{\tau} \cdot y_{\mathrm{eff}}}{v}$,

$u_{\tau}=\sqrt{\frac{\tau_{\mathrm{w}}}{\rho}}$.

In this case the preston tube to be used is additively manufactured to minimize its outer dimensions ensuring the measuring position to be well within the lower boundary layer. The preston tube has been installed flush mounted into a flat plate with no pressure gradient and a fully developed turbulent boundary layer. For speeds lower than $M a=0.3$, incompressibility can be assumed, such that the velocity $u_{p}$ at an effective heigth $y_{\text {eff }}$ can be expressed through the relationship shown in (17). The effective height which the net dynamic pressure corresponds to, lies just above the centerline and is due to the characteristic velocity profile at close proximity to the wall. This effect is being accounted for through the use of a displacement factor $K$.

$u_{P}=\frac{\sqrt{2 q}}{\rho}$,

$q=p_{t}-p_{s} ; \quad y_{\text {eff }}=d / 2 \cdot K ; \quad K=1.3$. 
Since the tubes outer diameter reaches up to areas beyond the viscous sub- and the buffer layer, the law of the wall in its complete form has to be accounted for. The van Driest law for adiabatic and fully turbulent flat plate boundary layers with a zero pressure gradient is defined as follows:

$u^{+}=\int_{a}^{b} \frac{2 \mathrm{~d} y^{+}}{1+\left[1+4\left(\kappa y^{+}\right)^{2}\left(1-\exp \left(-y^{+} / A^{+}\right)\right)^{2}\right]^{0.5}}$,

$\kappa=0.4 ; A^{+}=26$.

Combining the afore-mentioned relationships (1)-(6) one can utilise the simple computational preston tube method (CPM). Assuming an arbitrary initial value for $\tau_{P}$ leads to a pair of $u^{+}$and $y^{+}$. Only if those match the result of the numerical integrated van Driest Law for the derived $y^{+}$the correct wall shear stress is found. This procedure is being carried out for every measuring point, leading to the result shown in Fig. 7.

For verification reasons only, the calculated shear stress values $\tau_{P}$ are being transferred to their nondimensional form $\tau_{P}^{+}$. Plotting $q^{+}$over $\tau_{P}^{+}$results in the characteristic calibration curve for a preston tube, which was introduced by Patel (1965) and is shown in Fig. 8.

$\tau_{P}^{+}=\frac{\tau_{P} \cdot d^{2}}{4 \rho v^{2}}$,

$q^{+}=\frac{q \cdot d^{2}}{4 \rho v^{2}}$

The average deviation between the experimental data and the predicted Patel curve is about $2.8 \%$ proving the foregone calculation of $\tau_{P}$ to be valid and accurate. In compliance with (Nitsche et al. 1984) half the fence height is chosen to be the

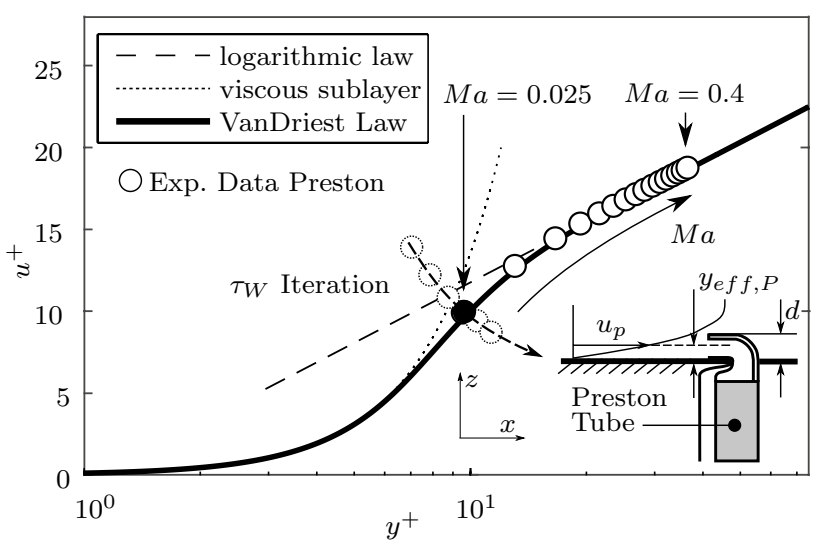

Fig. 7 Law of the wall for fully turbulent boundary layers and results from the CPM method

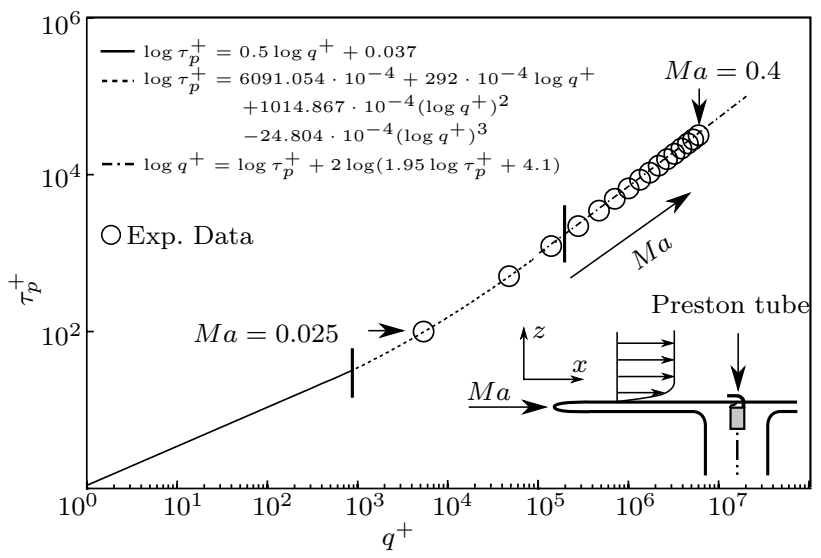

Fig. 8 Nondimensional wall shear stress models and corresponding CPM results

charactisitic wall distance as the fence is assumed to be fully immerged within the viscous sublayer. Such that the velocity $u_{F}$ at $h / 2$ can be derived by taking advantage of the similarity laws once more. Applying the calculated $\tau_{P}$ to the van Driest Law and solving the equation by numerical integration leads to the following relationship:

$u^{+}=f\left(y^{+}\right) \longrightarrow u_{F}\left(y=\frac{h}{2}\right)=u^{+} \cdot \sqrt{\frac{\tau_{P}}{\rho}}$.

Note: If the fence extends beyond the viscous sublayer, the linear relation between $u^{+}$and $y^{+}$is not being fulfilled anymore. Depending on the form factor $H$, the momentum distribution is then non-linear along the fence height resulting in a fence velocity that can no longer be assigned to the specific wall distance $h / 2$.

By applying the relation presented in 21 a direct combination of $\Delta p$ measured by the SFP and the extrapolated $u_{F}$ from the reference method can be achieved adequately. In order to appropriately account for density and temperature effects both quantities have been transferred into their nondimensional form $\Delta p^{+}$and $u_{F}^{+}$(see Fig. 9).

$\Delta p^{+}=\frac{\Delta p h^{2}}{4 \rho v^{2}}$,

$u_{F}^{+}=\frac{u_{F} h^{2}}{4 \rho v^{2}}$.

A logarithmic scale was introduced to allow for a linear regression function to the data, as it is commonly applied to wall friction calibrations of sublayer fences. The average deviation between the regression and the data obtained is about $0.33 \%$. Starting at a value of $\log \left(\Delta p^{+}\right)=7$, the experimental data appear to deviate increasingly from the calibration function. However, during measurements in the later presented compressor flow field $\log \left(\Delta p^{+}\right)$did not exceed 


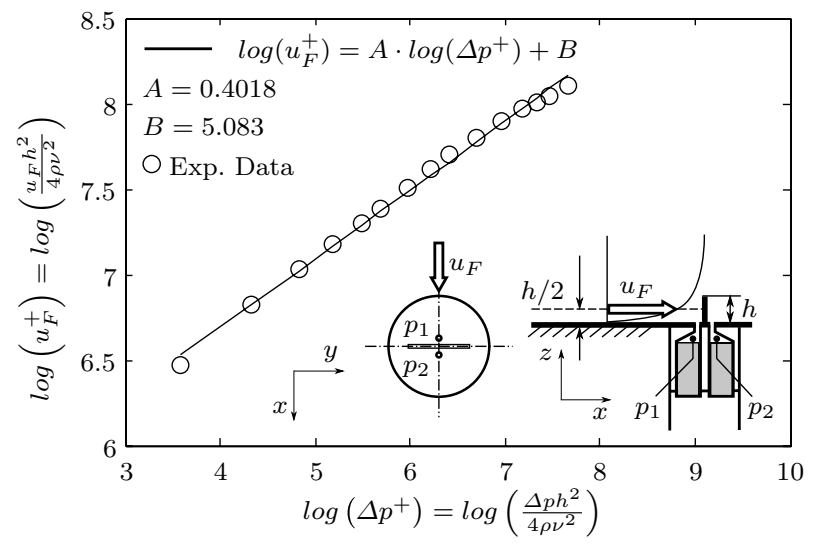

Fig. 9 Calibration curve of the SFP; normalized surface fence velocity against normalized pressure difference

values about 6.5 , hence, the non-linearity is of no concern within this work.

\section{Test section}

The second part of this paper reports on experimental investigations of an axial compressor cascade, conducted in a high speed wind tunnel at the Chair of Aero Engines at the Technische Universität Berlin. Here, the near wall flow field at the hub region has been observed using the aforementioned SFP.

The measurement domain of the test rig contains of a fully annular compressor stator cascade (CSC) with hub clearance. By its dimensions and blade dependent form factor, the cascade is representing a compressor stator from one of the last stages in a multistage axial compressor for jet engine applications. During operation of an axial compressor, the blades are exposed to different inflow angles resulting in varying aerodynamic loads. To appropriately simulate these conditions, a row of variable inlet guide vanes (VIGV) is positioned upstream. They generate swirl and thus set any desired inflow angle which the compressor blades are then exposed to further downstream. In the established nomenclature of compressor aerodynamics the inflow angle deviating from its design value is called "incidence angle" $i$ where positive incidence angles result in higher aerodynamic loads. The overall cascade dimensions are outlined in Fig. 10.

The radial clearance between the blade tips and the stationary hub wall is $3 \%$ span. Since the non-moving hub wall is not engine realistic, the Chen-criterion is introduced to check wether the boundary conditions at the hub are still representative:

$C_{\text {chen }}=s / c \sqrt{\operatorname{Re}} \geq 2.3$.

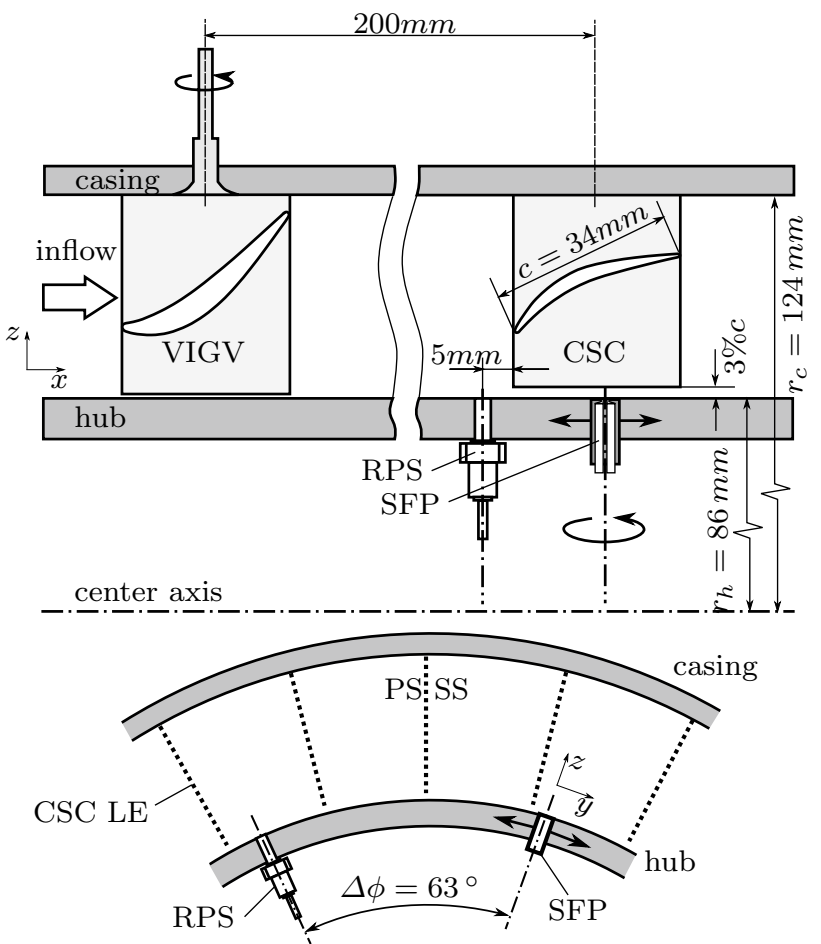

Fig. 10 Test section; dimensions of the high speed $360^{\circ}$ annular compressor stator and instrumentation

If the criterion is being fulfilled, the shear stress induced clearance velocity primarily caused by the relative movement between blades and hub wall is neglible compared to that velocity induced by the pressure difference between the blades pressure and suction side. During the experiments presented in this paper the Chen-criterion was about 18 at all times verifying the stationary hub wall to be appropriate for investigations on clearance related secondary flows.

The test rig has been part of extensive investigations over the last years. All of which concentrate on comprehending the complex secondary flow phenomena within the CSC. For this purpose different measurement techniques comprising oil flow visualization, five-hole probe measurements, hotwire measurements and time-resolved static pressure measurements on the side walls have been carried out (Beselt et al. 2012, 2013a, b, 2014; Pardowitz et al. 2012a, 2013, 2015; Peter et al. 2017). A detailed description of the inlet flow conditions to the CSC is provided by Beselt et al. (2014). The experiments yielded a flow phenomena of particulary interesting behaviour which has attracted significant attention within the scientific community. Specific properties of this phenomenon occur stochastically distributed in time at steady overall conditions of the compressor when it operates close to its aerodynamic stability limit. The overall operational conditions are stable, albeit a distinct reduction in pressure built-up. The flow phenomenon is known as a prestall instability, highly unsteady by nature 
Table 3 Specifications of reference pressure sensor (Kulite XT$190 \mathrm{M})$ flush mounted into the hub wall

\begin{tabular}{ll}
\hline Diameter & $3.8 \mathrm{~mm}$ \\
Pressure range & $5 \mathrm{PSI}$ \\
Full scale output (FSO) & $100 \mathrm{mV}$ \\
Non-repeatability & $0.1 \% \mathrm{FSO}$ typ \\
Resonance frequency & $150 \mathrm{kHz}$ \\
\hline
\end{tabular}

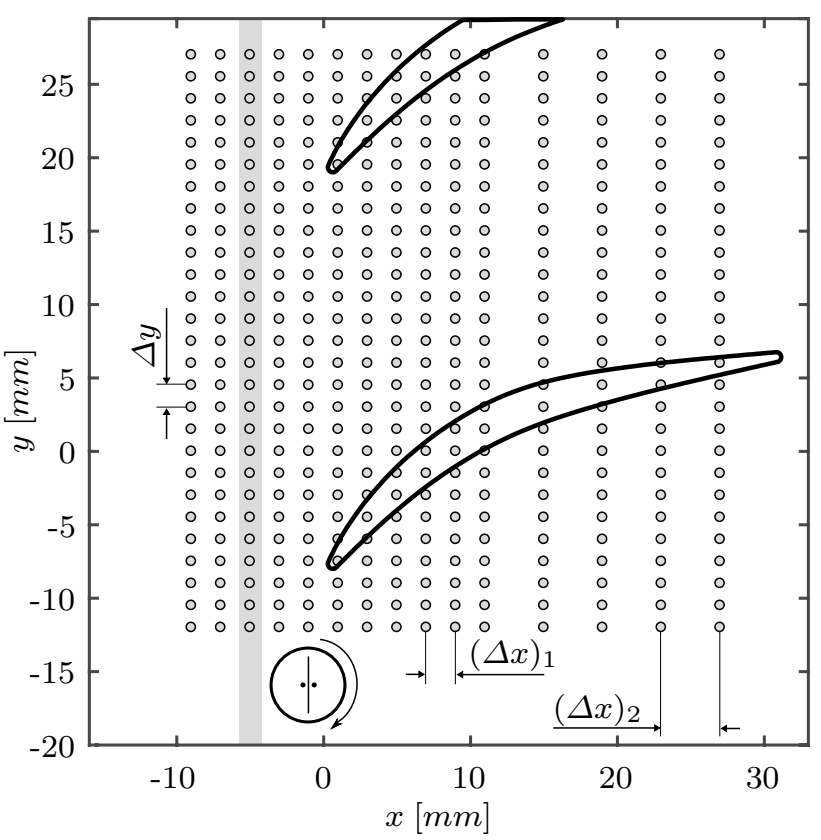

Fig. 11 Measurement locations of the SFP and axial position of the RPS (grey area)

and characterized by discrete disturbances propagating around the circumference of the cascade near the inlet plane $(-10 \mathrm{~mm}<x<0 \mathrm{~mm})$. Furthermore, these disturbances can be located in the vicinity of the tip clearance where the phenomenon is more likely to arise with larger tip gaps.

As the present work intends to further examine this specific type of prestall instabity an unsteady reference pressure sensor (RPS) has been flush mounted within the hub wall $5 \mathrm{~mm}$ upstream of the leading edges to detect the individual disturbances. Specifications of the RPS are summarized in Table 3.

Additionally, the SFP is installed inside the hub wall in order to measure the unsteady near wall flow field simultaneously. The SFP and the RPS are circumferentially separated by a constant $\Delta \Phi=63^{\circ}$, which corresponds to three and a half CSC passages. The entire hub, comprising both sensors, can be rotated underneath the CSC. Additionally the SFP can be be moved in axial direction. The resulting measuring grid extends over one and a half passages and $-10 \mathrm{~mm}$ up to $28 \mathrm{~mm}$ in axial range, see Fig. 11. All data were sampled at $20 \mathrm{kHz}$ with a hardware low-pass filter set at $10 \mathrm{kHz}$ to avoid aliasing.

Taking into account previous findings about the area of propagation of the prestall disturbances, the spatial density of the measuring positions has been increased near the cascade inlet plane. A traverse system installed inside the hub allows to rotate the SFP around its vertical axis $z$ in order to identify the flow direction. At all times during the measurements, the inflow velocity upstream the CSC is held constant at $c_{\text {inflow }}=135 \mathrm{~m} / \mathrm{s}$ corresponding to a chord based Reynolds number of $R e=350,000$. The resulting inflow Mach number is about $M a=0.4$.

\section{Mean flow field}

In order to choose a representative point of operation where the prestall instability is sufficiently pronounced the RPS measurements are being statistically analyzed. The basis to this are time-resolved reference pressures, recorded at various circumferential positions $\Phi$ and for different incidence angles in a range of $i=0^{\circ}$ (design point) up to $i=14^{\circ}$. Computing the second and the third statistical momentum at each operating point provides means to identify the inception of the prestall instability. The following equations have been used to calculate the root mean suare (RMS) and the skewness respectively:

$\operatorname{RMS}(t)=\sqrt{\frac{1}{N-1} \sum_{i=1}^{N}\left[p_{\text {ref }}(\Phi, t)-\bar{p}_{\text {ref }}(\Phi)\right]^{2}}$,

$\operatorname{skewness}(t)=\frac{1}{N} \sum_{1=1}^{N} \frac{\left[p_{\text {ref }}(\Phi, t)-\bar{p}_{r e f}(\Phi)\right]^{3}}{(\operatorname{RMS}(t))^{3}}$

Both parameters are depicted in Fig. 12 as a function of incidence $i$. It can be seen that the pressure fluctuation is at a low level between 1.6 and $1.8 \%$ of the dynamic pressure at the inlet until a sudden increase of RMS appears at $i=9^{\circ}$. Correspondingly the skewness significantly drops below zero from $i=9^{\circ}$ upwards indicating that the time-resolved static pressure is characterized by a higher number of pressure drops deviating significantly from the most common pressure value $\left(\operatorname{mode}\left(p_{\text {ref }}\right)\right)$. These discontinuities indicate the onset of prestall instability as it has been already demonstrated by Eck te al. (2017). The characteristic ramp-up in pressure fluctuations was previously described by Young et al. (2011) studying the blade pressure signature as a potential stall warning indicator.

The experimental investigations of both, the time averaged and the unsteady flow field focus on an operating point with distinct prestall instability. The data recording 


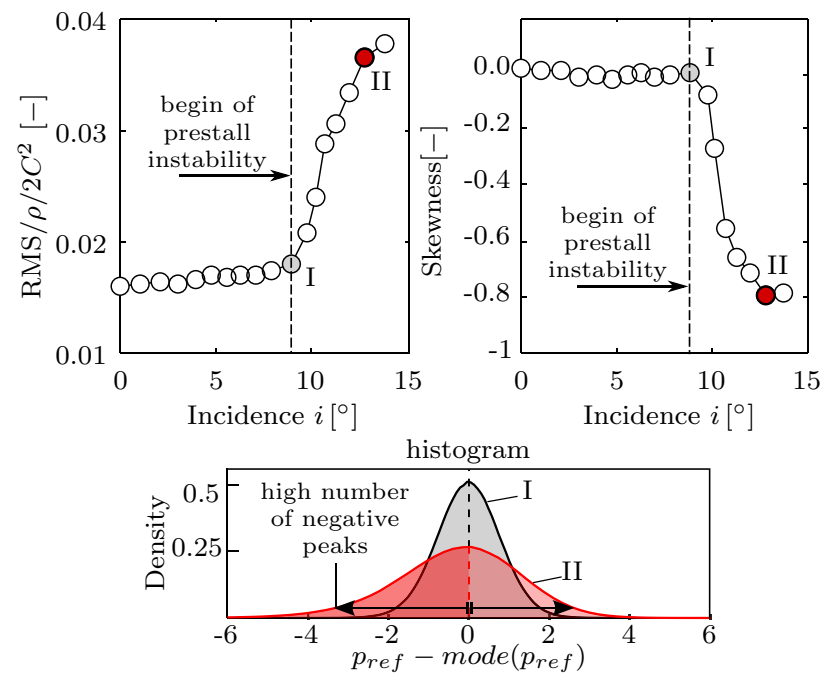

Fig. 12 Analysis of the reference pressure; normalized RMS and skewness against incidence $i$ and corresponding histogram for two selected operating points I and II

and analysis procedure of the SFP is made up of the following steps, resulting in the mean flow field at $i=12.8^{\circ}$ (highlighted by a red circle in Fig. 12).

1. Measure $\Delta p$ at each of the $18 \alpha$-positions $\left(\Delta \alpha=20^{\circ}\right)$ for 20 seconds, average it.

2. Plot $\Delta p=f(\alpha)$ over $360^{\circ}$, (cf. Fig. 4).

3. Apply the linearization method to the stepwise $\Delta p$-distribution (cf. Fig. 5).

4. Calculate $\beta$ and $\Delta p_{\max }$ based on Eqs. (7), (8910).

5. Compute the fence velocity $u_{F}$ using the calibration function in Fig. 9.

6. Repeat steps (1)-(4) at each grid location.
The resulting mean vector field is plotted in Fig. 13a. For a better interpretation of secondary flow structures the vector field is being transferred into a streakline pattern $b$ and then compared to a digitized flow pattern derived from oil flow visualizations (c).

The authors would like to point out some restrictions that must be considered when analyzing the results from the SFP. First of all, the SFP has not been calibrated in that environment it was later exposed to. Therefore, the displacement factor $K$ would have to be adjusted depending on whether the boundary layer is laminar or turbulent at each individual measurement point. Additionally, minor inaccuracies regarding the probes mounting inside the hub wall might affect the validity of the calibration function. Furthermore, the flow inside the stator cascade is highly three-dimensional whereby the boundary layer profile is significantly skewed in some areas and as such streamlines further above the wall may differ significantly from streaklines. Unfortunately, the latter effect cannot be compensated by any correction function. Measurements of static pressure contours on the hub wall by Beselt et al. (2014) revealed increased pressure gradients to be located along the pressure side of the blade. However, a correction or validation of the fence velocity was waived as Nitsche et al. (1984) and Nitsche and Brunn (2006) has confirmed that sublayer fences are relatively unsensitive to additional flow parameters like pressure gradient, heat flux and friction Mach number. Nonetheless, the authors would like to point out that the fence velocities may have unquantifiable errors, especially near the tip gap, since the boundary layer is thin in this area and the fence is most likely to reach out of the viscous sublayer. The potential error was discussed in Sect. 3. However, the area of interest lies upstream of the leading edge plane, thus far off from the tip gap.

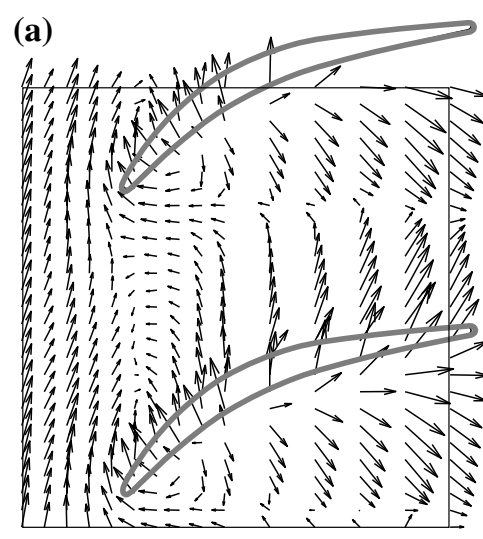

(b)

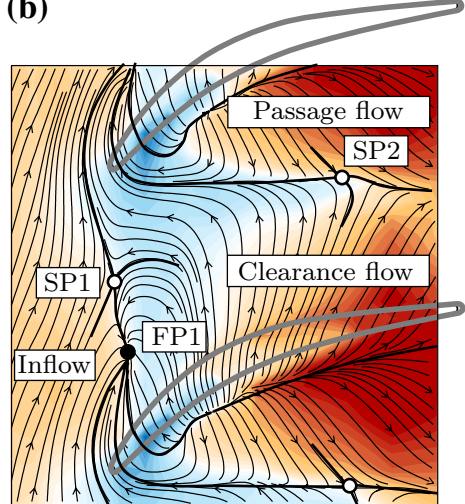

Axial velocity component $v_{F}[\mathrm{~m} / \mathrm{s}]$
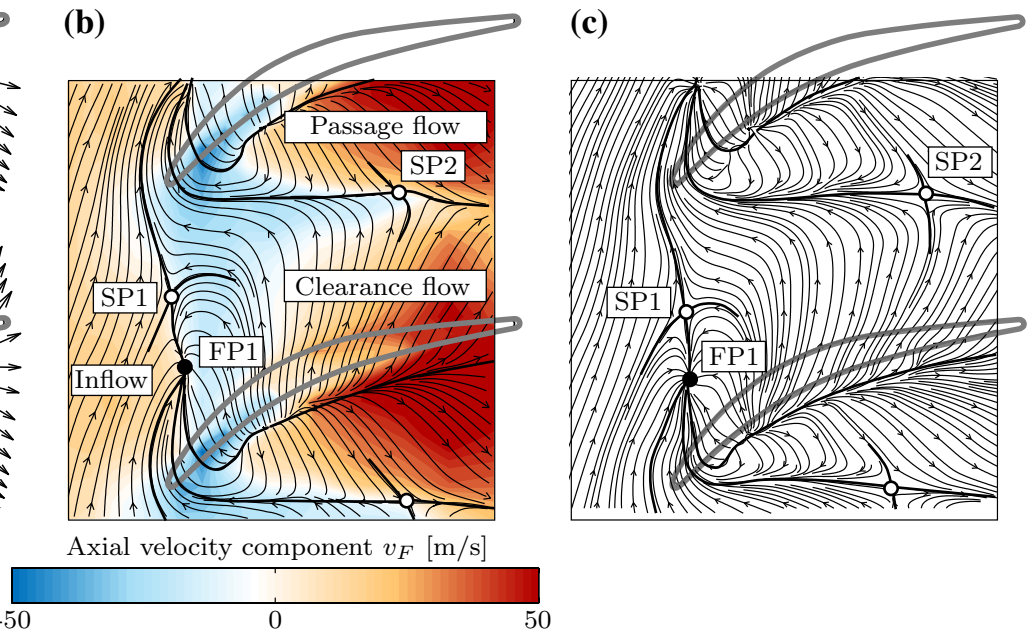
50

Fig. 13 Mean vector field of the sublayer flow field on the hub wall at $i=12.8^{\circ}(\mathbf{a})$; corresponding streakline pattern of the secondary flow structures (b); digital streakline pattern derived from oil flow visualizations (c) 
In result, experimental streakline patterns are being generated, both time-averaged and -resolved. They represent a flow visualization tool which in this detail was previously limited to computational fluid simulations and allow for qualitative interpretation of the adjoined boundary layer.

The pattern shown in Fig. 13b, c are in excellent compliance validating the underlying measurement principle. The computed flow direction coincide with the direction of the wall shear stress lines, derived from the oil flow visualization. Even the location of flow singularities, namely spiral focuses (FP) and saddle points (SP) are being captured by the traversed SFP. Minor differences are recognized in the vicinity of separation lines where the flow is characterized by high directional velocity gradients. These gradients can only be captured up to a certain amount since the integral velocity vector is being averaged over an area defined by the length of the fence. Hence the slight deviation to the oil flow patterns.

As a whole, the flow field inside the passage is dominated by the clearance flow colliding with the passage cross flow which is driven by the pressure gradient between the blades pressure side (PS) and the suction side (SS). A large amount of clearance flow is redirected towards the cascade inlet plane, in opposition to the intended main flow direction and spills around the leading edge of the neighboring blade. "Forward spillage" is known as a characteristic flow feature near the stability limit of compressor cascades and it is ascribed to be a prominent trigger for rotating stall.

The color-coded axial velocity component in Fig. 13b demonstrates the reversed flow extending over the whole circumference at the passage inlet. As such, the clearance flow acts like a blockage enforcing the incoming boundary layer to separate from the hub wall before entering the cascade.

\section{Unsteady flow field}

The very intention of the instationary streakline pattern is to give insights to the present prestall instability. Thus, revealing the flow pattern responsible for the RMS ramp-up and low pressure disturbances in a compressor passage dominated by forward spilled clearance flow. To begin with, the ability of the measurement system to capture these disturbances, has to be verified. This is done by applying a power spectral density (PSD) to the measurement data obtained by the RPS and the SFP each located $5 \mathrm{~mm}$ upstream of the leading edges. For comparison reasons, the PSD spectra are being normalized with their corresponding, grid-point specific variance, a measure of the average power of the signal. The PSD is defined as follows:

$\operatorname{PSD}=\frac{2}{T}\left|p\left(f_{m}\right)\right|^{2}$, where,

$p\left(f_{m}\right)=A_{m}(f)=\Delta t \sum_{n=0}^{N-1} p\left(t_{N}\right) \mathrm{e}^{-i 2 \Pi \frac{m n}{N}}$,

represents the fourier transformation of the pressure signal. The RPS spectrum in the top part of Fig. 14, shows a distinctive spectral signature comprising a broad banded chain of peaks between 100 and $500 \mathrm{~Hz}$ which arise at the incidence angle from which the RMS also increases and the skewness starts showing negative values respectively (cf. Fig. 12). This behaviour was previously observed in similar experiments by Eck te al. (2017). At this point, it is important to mention that this very PSD signature represents a reliable way of detecting a phenomena called "Rotating Instability" (RI) (Baumgartner et al. 1995; Kameier and Neise 1997; Mailach et al. 2001; März et al. 2002; Beselt et al.
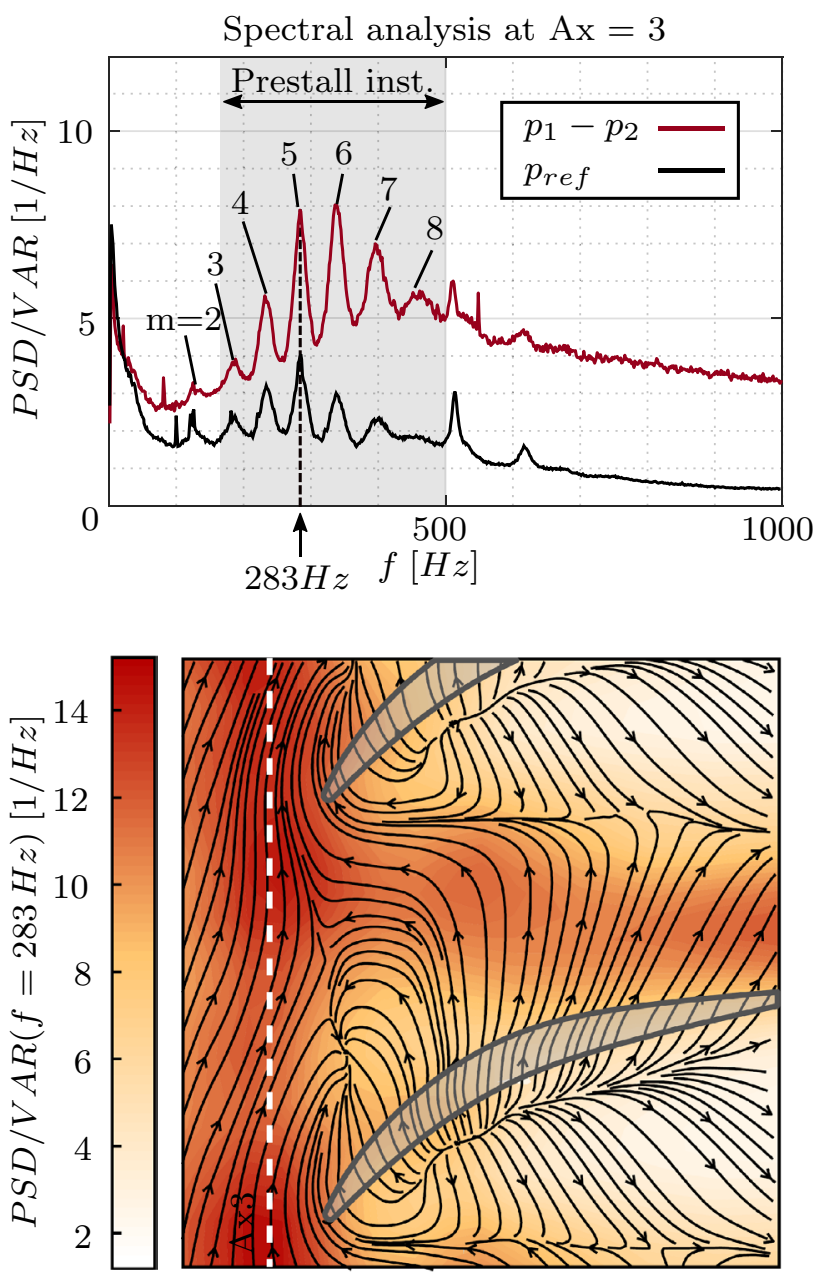

Fig. 14 Spectral analysis for $i=12.8^{\circ}$; identification of the propagation area of the prestall instability by analyzing the spectral density at a frequency of $f=283 \mathrm{~Hz}$ 
2013a). Comprehensive studies of RI in this very application revealed that each peak within the characteristical signature can be assigned to one specific circumferential mode order $m$. Further investigations demonstrated that the mode order depends on the number of flow disturbances equally distributed around the circumference. The propagation speed of these disturbances can be calculated from the product of the difference frequency between the adjacent peaks and the hub circumference (Pardowitz et al. 2012a).

Additionally, the power spectrum from the time-resolved pressure difference $\Delta p$ of the SFP is plotted in the top part of Fig. 14. Again, the signature arises around $300 \mathrm{~Hz}$ proving the physical output of the SFP is capable of resolving the high frequency velocity fluctuations linked to the prestall disturbances. Note, the thin peak at $510 \mathrm{~Hz}$ is ascribed to an accoustic axial mode of the test facility itself as elaborated by Pardowitz et al. (2012b). In a first attempt to narrow down the area of particulary energetic fluctuations associated with the present prestall instability, the PSD of the SFP has been carried out at each grid location. Only the amplitude values for one specific frequency, $f=283 \mathrm{~Hz}$ (cf. Fig. 14, bottom), have been plotted. High magnitudes of the PSD at the leading edge plane give a first indication of the prestall disturbances to develop along the separation line and that some part of that higher energy fluid is being transported downstream inside the passage.

In the following, the flow structures associated with those fluctuations shall be observed from a vector field point of view. Every time a prestall disturbance passes through, the SFP shall be read out and provide information about the flow direction and its absolute value. As these disturbances are associated with distinctive negative pressure spikes, first mentioned in Sect. 5, the latter will serve as a trigger to define read out times for the SFP. They will be looked for in the RPS signal. At this point the purpose of the RPS becomes clear. Whereas the SFP varies in location within the grid the RPS holds its axial position at every time, ensuring consistent and equal trigger intensities. Since both sensors are synchronized in time, a propagating flow disturbance the RPS is exposed to will also be detected by the SFP in a change of velocity. In short, every time a negative pressure spike is detected via the RPS, the SFP's $\Delta p$ values are read out, and ensemble averaged afterwards.

A series of detected triggers in the unfiltered RPS signal is exemplarily shown in Fig. 15 and marked by red triangles. A trigger is defined by the pressure dropping below a defined threshold level whereby the latter is being adjusted by incremental steps until at least 100 triggers have been identified. Additionally, a minimum distance between the individual triggers was predefined to avoid the sequences to overlap. Figure 15 demonstrates how the pressure spikes appear stochastically distributed in time. The exemplary cut-out of the signal in Fig. 15, bottom contains two triggers each of which

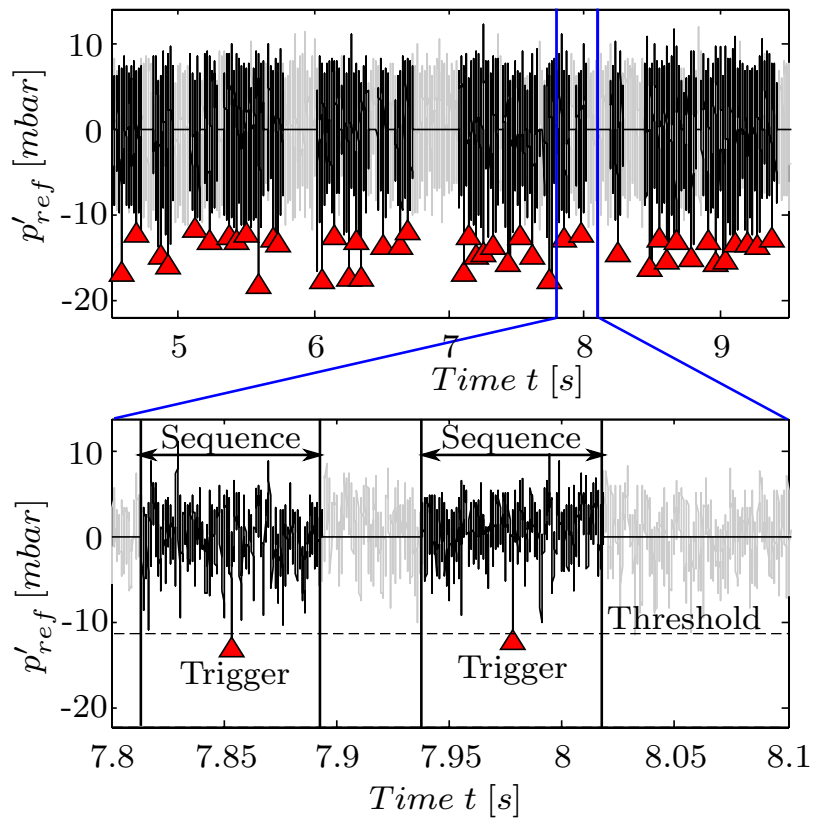

Fig. 15 Detemination of stochsatic trigger times for sequence averaging (highlighted by red triangles)
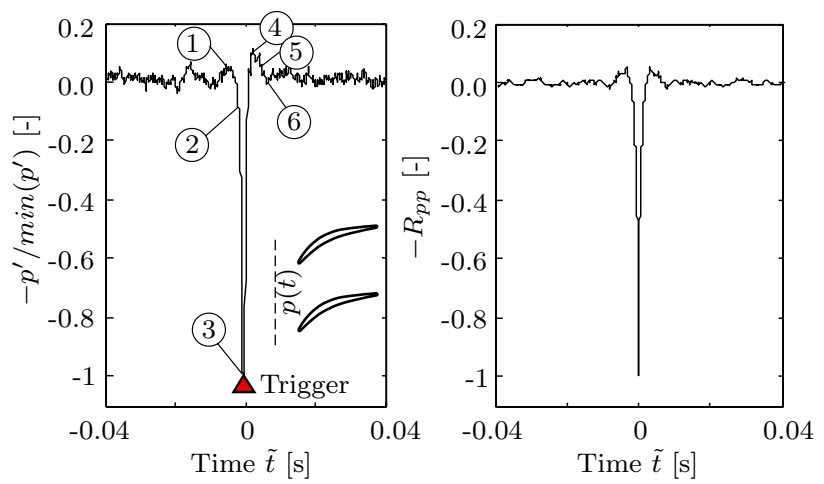

Fig. 16 Ensemble-averaged sequence (left) and auto-correlation function of reference pressure (right); the numbering indicates the points in time at which the unsteady flow field is shown in Fig. 17

specify the center-location of the pressure sequences with a predefined length of $\Delta \tilde{t}=8 \mathrm{~ms}$. The corresponding ensemble averaged sequence shows a distinct negative spike enclosed by harmonic amplitude overshoots (Fig. 16, left). Applying an auto-correlation to the raw RPS signal of length $t=20 \mathrm{~s}$ leads to the same characteristic. Since the auto-correlation is a common tool for identifying repeating patterns in a signal, the correlation coefficient itself is mirroring the recurring flow property present in this particular case. If the signal contained noise only, the autocorrelation would comprise a single peak in the centre without the harmonic overshoots.

The propagation speed of the spike in circumferential direction, can be determined by carrying out a cross correlation of the RPS's $p_{\text {ref }}(t)$ and the SFP's $\Delta p(t)$. The respective 
value is $k=31 \mathrm{~m} / \mathrm{s}$ being consistent with the propagation speed derived from the spectral signature in Fig. 14.

In summary, the data analysis process to obtain the phase averaged time resolved vector flow field consists of the following steps:

1. Find 100 trigger within the RPS signal for each rotation angle $\alpha$ of the SFP, save the $\Delta p$ sequence $\pm 4 \mathrm{~ms}$ around the trigger $\tilde{t}=0 \mathrm{~ms}$

2. Ensemble average the 100 sequences such that at every $\alpha$ there is a $\Delta p=f(\tilde{t})$.

3. Extract the $\Delta p$ distribution (see Fig. 3) for a constant $\tilde{t}$ and all 18 rotational positions of the SFP.

4. Compute the velocity and flow angle for every $\Delta p(\alpha, \tilde{t}=$ const $)$ for $\alpha=\left\{0^{\circ} \ldots 20^{\circ} \ldots 340^{\circ}\right\}$ by executing the stepwise procedure presented in chapter 5 .

5. Repeat step (4) for every $\tilde{t}$.

6. Perform the previous steps at each grid point within the passage.

In result, the entire vector field can be plotted within the timeframe $\tilde{t} \in\{-4 \mathrm{~ms} \cdots+4 \mathrm{~ms}\}$ when the disturbance is present within the passage (indicated by no. 1-6 in Fig. 16).

For a better understanding of the underlying flow structures a classical reynolds decomposition has been carried out, which allows for in depth analysis of the fluctuating term exclusively:

$\vec{c}(x, y, \tilde{t})=\overline{\vec{c}}(x, y)+\vec{c}^{\prime}(x, y, \tilde{t})$

where the mean velocities at each grid point have already been derived in Sect. 5. The result, a chronological sequence of the vector field is shown in Fig. 17. In addition, a vortex identification method, namely the Q-criterion, was superimposed in order to better identify emerging coherent flow structures. To compute $\mathrm{Q}$, the velocity gradient tensor was decomposed into its symmetric and anti-symmetric part, where the second invariant of the gradient tensor can be expressed as follows:

$Q=\frac{1}{2}\left(\Omega_{i j} \Omega_{j i}+S_{i j} S_{j i}\right)$,

where,

$\Omega_{i j}=\frac{1}{2}\left(\frac{\partial c_{i}}{\partial x_{j}}-\frac{\partial c_{j}}{\partial x_{i}}\right)$

$S_{i j}=\frac{1}{2}\left(\frac{\partial c_{i}}{\partial x_{j}}+\frac{\partial c_{j}}{\partial x_{i}}\right)$

are known as the vorticity tensor and the rate-of-strain tensor respectively. A vortex has been found, if the vorticity
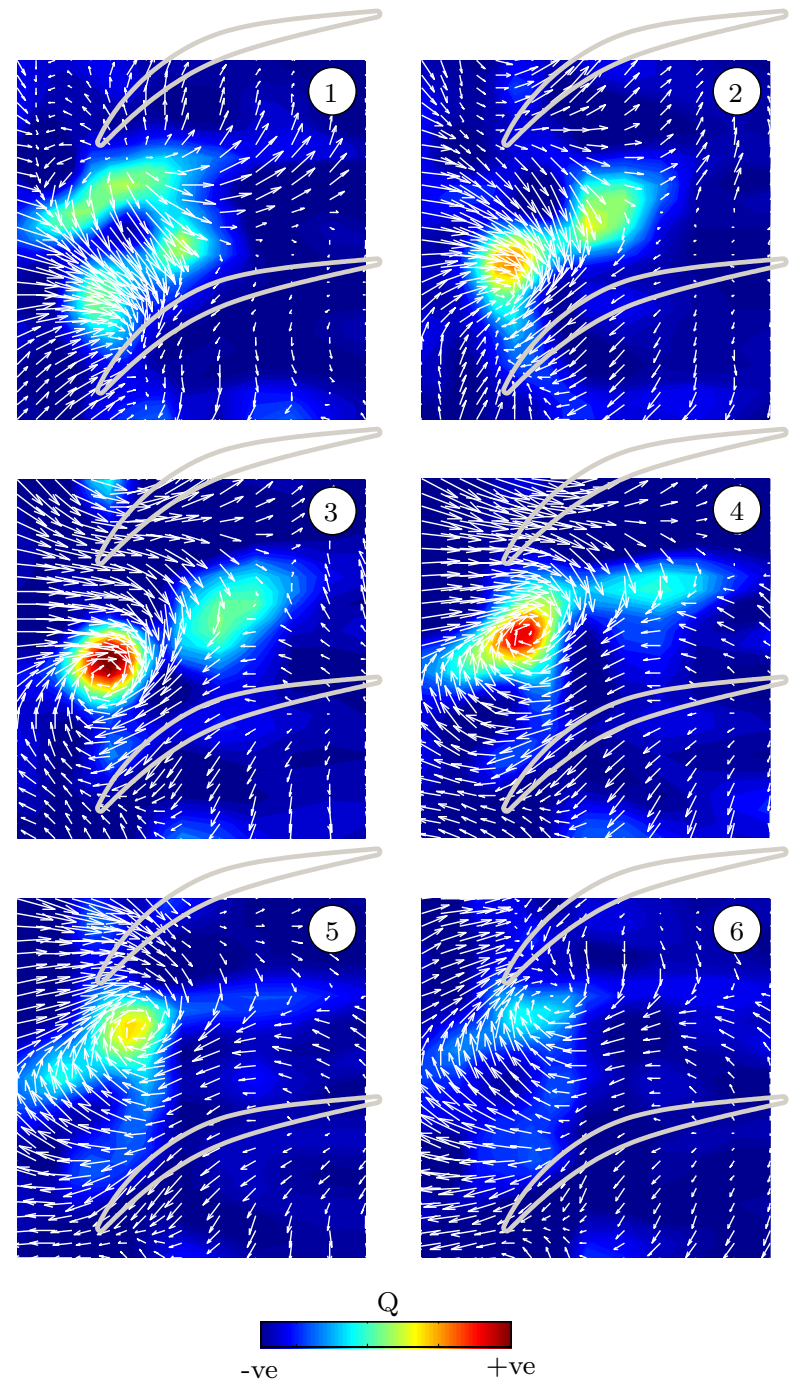

Fig. 17 Ensemble averaged flow field associated with the trigger time; detection of vortex structures based on Q-criterion

magnitude is greater than the shear strain rate. Thus, where $\mathrm{Q}$ takes positive values.

Based on the velocity conventions defined in this paper, Eq. (30) can be transferred into the following expression:

$Q=-\frac{1}{2}\left(\frac{\mathrm{d} v^{2}}{\mathrm{~d} x^{2}}+\frac{\mathrm{d} w^{2}}{\mathrm{~d} y^{2}}\right)-\frac{\mathrm{d} v}{\mathrm{~d} y} \frac{\mathrm{d} w}{\mathrm{~d} x}$.

Looking at the resulting images, the highest level of fluctuations is to be observed in the leading edge plane which is in good agreement with the frequency analysis in Fig. 14. The vector field at $\tilde{t}=0 \mathrm{~ms}$ illustrates that the pressure spike is linked to a clockwise rotating vortex structure which can also be distinguished by increased $Q$ values. Considering the previous and subsequent points in time, the vortex trajectory continues along the separation line located upstream of the 
passage. The coherent flow structure is evidently changing its vorticity while moving in circumferential direction. In general, the propagation area is in good agreement with previously observed pathes of intense low pressure which could be identified in the course of investigations on prestall flow disturbances (Inoue et al. 2004; Weichert and Day 2013; Young et al. 2011; Eck et al. 2015). Furthermore, the vortex induces an alternating acceleration and deceleration of the flow inside the passages.

Note, the extracted information yields a phase averaged flow field only. Also, the trigger method used here entails, that only the pressure drops with highest amplitudes are being taken into account. That would be the recurring pressure fluctuation responsible for the amplitude peak at $283 \mathrm{~Hz}$ in Fig. 14. This also implies that pressure drops surrounding the triggered spikes with varying circumferential displacements and thus being responsible for different mode orders are going to be averaged out.

In conclusion, it can be stated that the measurement principle is capable to identify the secondary flow structure underlying the dominant feature of this specific prestall instability. For the first time, the often proposed idea of radially aligned, tornado like vortices inducing localized low pressure spots at the adjoining endwall has been experimentally confirmed. Further analysis will help to give insights into what flow mechanisms are involved behind the formation of the disturbance by extending the field of view beyond single passage analysis.

One question which arises is, to what extend the structure influences neighboring passages and whether the dimension of the disturbance is of rather short- or long-length scale. By knowing the propagation speed of the disturbance, a quasiinstantaneous, multi passage vector field can be build over any desired portion of the circumference. A special sorting algorithm allows to select single passage vector fields from the sequence and transfer the included information through a time shifting procedure to a neighbouring passage, each with a circumferential offset of one pitch. The individual vector fields are then being appended to each other. As a result, the generated multi-passage plot allows for proper farfield inspections also over the course of time. (The described sorting algorithm can be found in Eck et al. (2015) in more detail).

The result of the quasi-instantaneous flow field is shown in Fig. 18, where the trigger vortex is clearly visible in passages (3-4). The image also shows the substantial reduction of velocity fluctuations within the neighboring passages. Furthermore, one can see an un-equidistantly arrangement of weaker anti-clockwise rotating vortices in passages (1-2). Hence, the acceleration area is much smaller in size compared to the region affected by deceleration. Subsequently, it will be shown that this specific arrangement of coherent structures induce a rather familiar flow pattern.

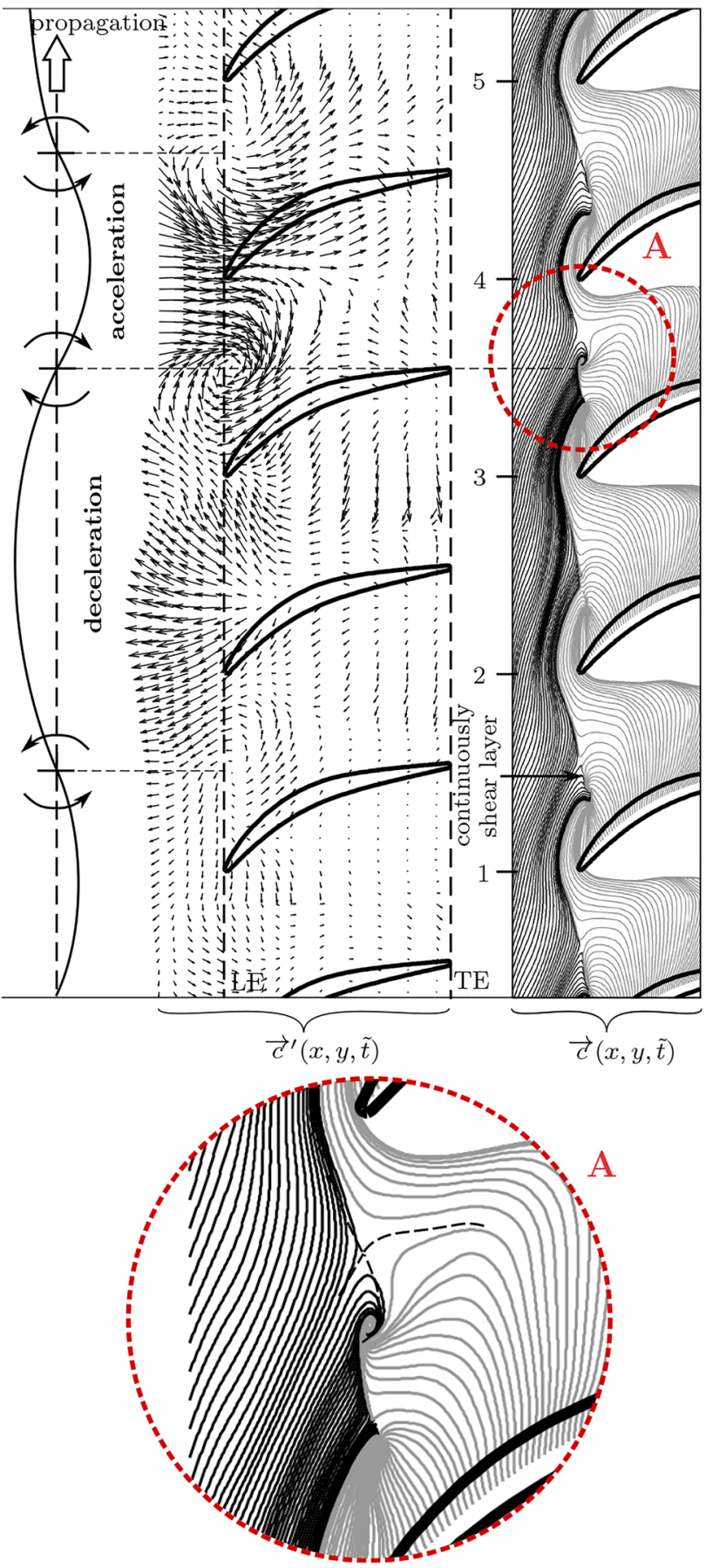

Fig. 18 Quasi-instantaneous plots of $\vec{c}$ and $\vec{c}^{\prime}$ (see Eq. 29) over a quarter circumference of the CSC; development of a short-length scale disturbance in the interface between the incoming main flow and the reversed clearance flow

For that purpose Fig. 18 shows the corresponding absolute streakline pattern, calculated off the $\vec{c}$ distribution. To easier distinguish between different parts of near wall passage flow, namely divided into main-inflow and clearance portion, the streaklines originating in the tip gap are colored 
differently from the ones that originate in the upstream inlet plane. By doing so, it becomes clear that the entire inflow boundary layer on the hub wall is prevented from entering the cascade at any passage or at any time by the forward spilling clearance flow. Again, a coherent flow structure, corresponding to the clock-wise rotating vortex identified in the fluctuation field, is clearly visible near the interface where the two flow parts converge.

Similar radial vortices have been observed previously. Those are assigned to the spike stall inception type. They are initially caused by a leading edge separation and they transition into a stall cell whithin a short period of time. Furthermore, there is strong evidence that they arise first and induce forward spillage afterwards. In contrast, in the present work forward spillage is observed in every passage albeit the coherent structures only occur in specific ones. Hence, those disturbances cannot be the underlying mechanism of spillage in this particular case. Spike stall inception and prestall disturbances are evidently to different flow phenomena. One hypothesis about the inception mechanism is based uppon the reasonable assumption that a shear layer which is undoubtedly present at the inlet of the passage becomes unstable and subsequently leads to the formation of small disturbances. Nevertheless, the actual inception mechanism of the herin observed coherent prestall flow disturbances cannot be clarified unambiguously.

Now, in contrast to Fig. 18, multi-passage view shall be generated for arbitrary time-steps before and after the actual trigger time. In order to do so, the starting point for the sample shifting algorithm is successively being delayed by one sample. Taking into account the sampling frequency of $s f=20 \mathrm{kHz}$, then 357 samples $(20,000 \mathrm{~Hz} / 56 \mathrm{~Hz})$ have been recorded while the disturbance propagated along the circumference. This results in about 18 samples per passage (357 samples/20 passages) which in turn corresponds to the maximum time resolution of the multi-passage vector field. In Fig. 19 only every third time step is being displayed. As additional information, the scalar divergence field is colorcoded in the background. The divergence can be interpreted as a way of indicating wether fluid is entering or leaving the plane upon which the vector field is measured. In other words, if the divergence is positive, fluid is attaching to the wall and vice versa. Its mathematical definition is as follows:

$\operatorname{div} \vec{F}=\frac{\partial v}{\partial x}+\frac{\partial w}{\partial y}$.

Negative values of the divergence indicate a sink where the streamlines converge into a spiral focus and transports fluid away from the wall. In contrast sources are defined as areas of positive divergence levels. Introducing the nomenclature of flow singularities, sinks are usually referred to as stable singularities and sources are known as to be unstable. It is clearly visible in Fig. 19 that the trigger vortex is represented by a stable singularity which is enclosed by two unstable singularities - all of them lined up along the leading edge plane. Obviously there is not only an alternating acceleration and deleleration of the flow in axial direction but also a periodical oscillation of the flow in radial direction. Generally, this particular arrangement of singularities strongly reminds of a model describing the early stage of flow instabilities. The latter can be described as a superposition of a stratified shear flow and a specific vorticity distribution along the respective interface with an alternating arrangement of stable and instable singularities (Carpenter et al. 2011). This agreement supports the theory about prestall flow disturbances being the result of a shear layer located at the passage inlet which becomes unstable with time.

The authors want to stress the fact once more, that the shown results are far off from real life flow patterns one would find when looking at global instantaneous vectorfields. Rather than just one pronounced vortex, there would be many, of varying strength, distributed irregularly around the circumference, affiliated to different azimuthal modes, and with varying convection speeds. The phase averaging procedure simplifies the flow pattern by a significant amount to allow primary and qualitative interpretations of one discrete disturbance associated with "Rotating Instability".

\section{Conclusions}

A new algorithm to evaluate surface fence probe data has been developed and applied. This allowed for implicitly measuring near wall flows both directional and in regards to its absolute velocity. The probe was deployed in a high speed wind tunnel predominantly used for investigations on secondary flows in axial compressors for aero engines.

Mean vector fields derived from the sublayer fence probe data have been validated by comparing them to digitized streakline patterns based on oil flow visualizations. The time-resolved vector fields taken at aa operating point close to the stalling limit revealed coherent flow structures which are affiliated with a specific type of prestall instability. The general findings of the present work can be summarized as follows:

1. To determine the flow angle of a specific near wall flow a surface mounted SFP is rotated stepwise around its vertical axis. An improved analysis method was presented which minimizes the number of required rotational steps while increasing the accuracy in flow angle calculation at the same time.

2. Using a reference calibration principle, namely the preston tube method, the pressure difference measured by the SFP could be linked to a flow velocity at half of the fence height. 

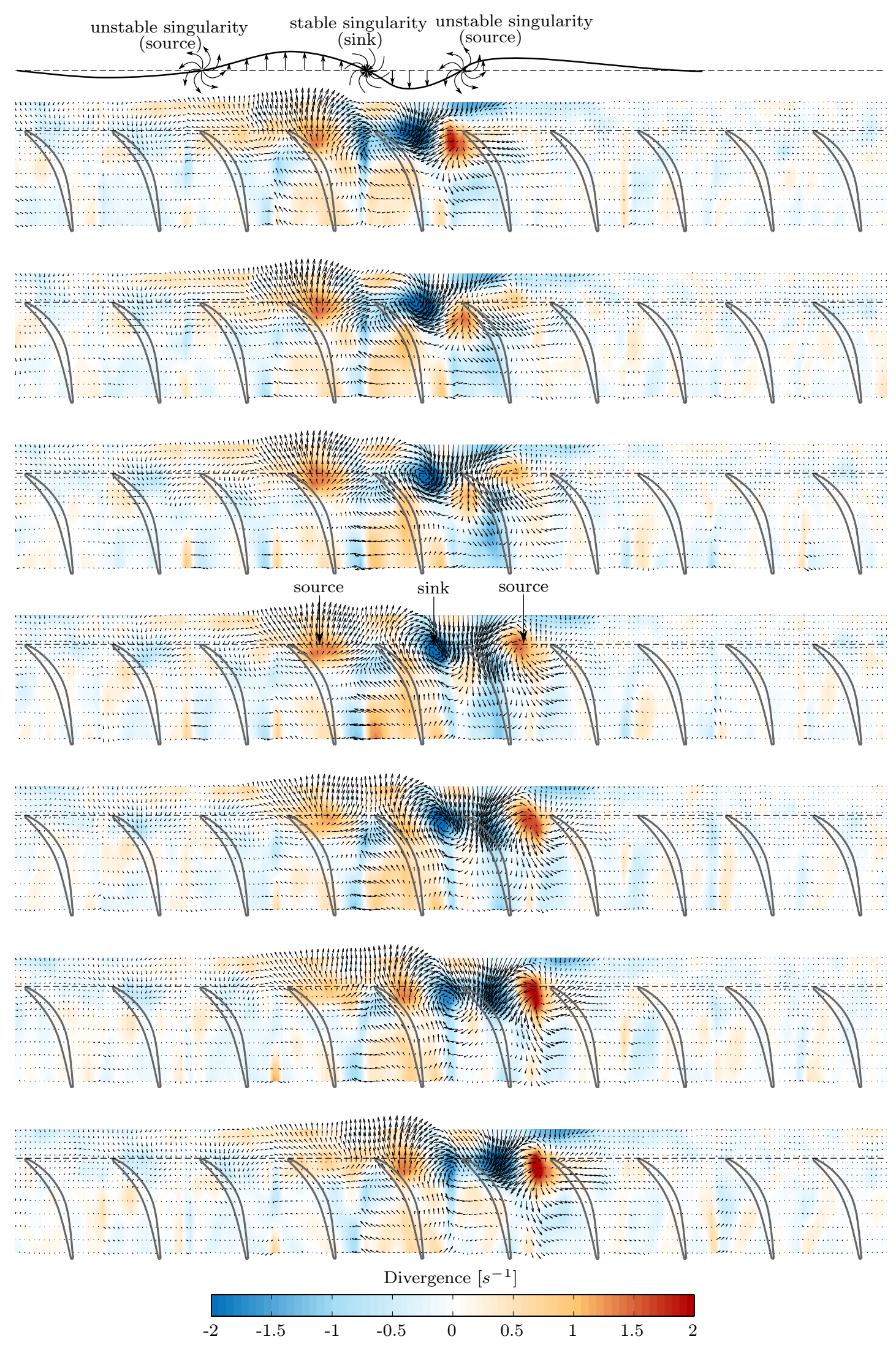

Fig. 19 Divergence for identification of sinks and sources within the field of velocity flucuations 
3. Measurements of time-resolved properties of near wall flows were made feasible by using miniature unsteady pressure sensors being embedded in the SFP. The line cavity system connecting the static pressure ports and the transducer has a natural frequency of $3390 \mathrm{~Hz}$ which is about 11 times as high as the frequency to be resolved in the flow field of concern. Hence, no damping function needed to be applied to the data aquired by the probe. Additionally a special sorting algorithm has been developed that uses recurring stochastic properties of the flow as a trigger for recomposing an ensemble averaged 2D contour of velocity fluctuations in magnitude and direction. By this, time-resolved oil flow picture equivalent to streakline patterns could be experimentally investigated to this detail for the first time.

4. The primary purpose of employing the SFP was to understand the highly three-dimensional secondary flow in a fully annular compressor stator cascade with hub clearance by visualizing the boundary layer flow on the hub wall. The operating point of the stator cascade was set to near stall conditions where a recently documented type of prestall instability appears. The latter is characterized by low pressure spikes propagating in the vicinity of the leading edge plane. In order to visualize the spike related flow topology the SFP data has been read out and evaluated at the very points in time where those spikes become apparent.

5. It was found that the pressure spikes are related to vortex structures travelling along a shear layer. They arise in the interface between the main flow and the reversed clearance flow. An alternating arrangement of stable and unstable singularities was recognized in the vector field of velocity fluctuations denoting the flow disturbances might be caused by a shear layer instability.

Open Access This article is distributed under the terms of the Creative Commons Attribution 4.0 International License (http://creativeco mmons.org/licenses/by/4.0/), which permits unrestricted use, distribution, and reproduction in any medium, provided you give appropriate credit to the original author(s) and the source, provide a link to the Creative Commons license, and indicate if changes were made.

\section{References}

Baumgartner M, Kameier F, Hourmouziadis J (1995) Non-engine order blade vibration in a high pressure compressor. In: Twelfth international symposium on airbreathing engines, Melbourne, Australia. https://hal.archives-ouvertes.fr/hal-01353829, https://hal.archivesouvertes.fr/hal-01353829/file/isabeb.pdf

Beselt Ch, van Rennings R, Thiele F, Peitsch D (2012) Experimental and numerical investigation of rotating instability phenomenon in an axial compressor stator. In: 42nd AIAA fluid dynamics conference and exhibit 2012. https://doi.org/10.2514/6.2012-2980
Beselt Ch, Pardowitz B, van Rennings R, Sorge R, Peitsch D, Enghardt L, Thiele F, Ehrenfried K, Thamsen P-U (2013a) Influence of the clearance size on rotating instability in an axial compressor stator. In: Proceedings of 10th European turbomachinery conference, No. ETC2013-141, Lappeenranta, Finland, 15-19 April 2013

Beselt C, Peitsch D, van Rennings R, Thiele F (2013b) Impact of hub clearance on endwall flow in a highly loaded axial compressor stator. In: ASME Turbo Expo 2013: turbine technical conference and exposition, number GT2013-95463. San Antonio (3-7 June 2013)

Beselt Ch, Eck M, Peitsch D (2014) Three-dimensional flow field in highly loaded compressor cascade. J Turbomach 136(10):10 (101007)

Beselt C, Peitsch D, van Rennings R, Thiele F, Ehrenfried K (2014) Experimental and numerical investigation of the unsteady endwall flow in a highly loaded axial compressor stator. In: ASME Turbo Expo 2014: turbine technical conference and exposition, Düsseldorf, Germany, 16-20 June 2014 (number GT2014-25944)

Carpenter JR, Tedford EW, Heifetz E, Lawrence GA (2011) Instability in stratified shear flow: review of a physical interpretation based on interacting waves. Appl Mech Rev 64:11

Dambach R, Hodson HP (1999) A new method of data reduction for single-sensor pressure probes. Proc ASME Turbo Expo. https:// doi.org/10.1115/99-GT-304

Eck M, Geist S, Peitsch D (2017) Physics of prestall propagating disturbances in axial compressors and their potential as a stall warning indicator. Appl Sci 7(3):285

Eck M, Tiedemann C, Peitsch D (2015) Detailed measurements of the unsteady pressure field in the area of origin of rotating instability. In: ISUAAAT 14, No. I14-S3-3

Inoue M, Kuroumaru M, Yoshida S, Minami T, Yamada K, Furukawa M (2004) Effect of tip clearance on stall evolution process in a low-speed axial compressor stage. https://doi.org/10.1115/GT200 4-53354

Kameier F, Neise W (1997) Experimental study of clearance losses and noise in axial turbomachines and their reduction. $\mathrm{J}$ Turbomach 119:460-471

Mailach R, Lehmann I, Vogeler K (2001) Rotating instabilities in an axial compressor originating from the fluctuating blade tip vortex. J Turbomach 123:453-463

März J, Hah C, Neise W (2002) An experimental and numerical investigation into the mechanisms of rotating instability. J Turbomach 124:367-374

Nitsche W, Brunn A (2006) Strömungsmesstechnik, VDI-Buch., 2nd edn. Springer, Berlin, Heidelberg

Nitsche W, Haberland C, Thuenker R (1984) Comparative investigations on friction drag measuring techniques in experimental aerodynamics. In: 14th Congress of the International Council of the Aeronautical Sciences, number ICAS-84-2.4.1

Nitsche W, Thünker R, Haberland C (1985) A computational preston tube method. In: Bradbury LJS, Durst F, Launder BE, Schmidt FW, Whitelaw JH (eds) Turbulent shear flows 4. Springer, Berlin, Heidelberg, pp 261-276

Pardowitz B, Tapken U, Enghardt L (2012a) Time-resolved rotating instability waves in an annular cascade. https://doi. org/10.2514/6.2012-2132 (ISBN 978-1-62276-215-6)

Pardowitz B, Tapken U, Enghardt L (2012b) Acoustic resonances and aerodynamic interactions in an axial compressor stator stage test rig (ISBN 978-095-485-8346)

Pardowitz B, Tapken U, Sorge R, Thamsen P-U, Enghardt L (2013) Rotating instability in an annular cascade: detailed analysis of the instationary flow phenomena. ASME J Turbomach. https:// doi.org/10.1115/GT2013-95820

Pardowitz B, Peter J, Tapken U, Thamsen P-U, Enghardt L (2015) Visualization of secondary flow structures caused by rotating instability: synchronized stereo high-speed PIV and unsteady pressure measurements. https://doi.org/10.2514/6.2015-2930 
Patel V (1965) Calibration of preston tube and limitations of its use in pressure gradients. J Fluid Mech 23:185-208

Peter J, Pardowitz B, Eck M, Enghardt L, Peitsch D, Thamsen PU (2017) Advanced stereo high-speed PIV in an annular cascade without clearance: evidences of rotating instability. In: Proceedings of 12th European conference on turbomachinery fluid dynamics and thermodynamics. https://elib.dlr.de/116951/

Schober M, Obermaier E, Pirskawetz S, Fernholtz H-H (2004) A mems skin-friction sensor for time resolved measurements in separated flows. Exp Fluids 36:593-599

Vagt JD, Fernholtz H (1973) Use of surface fences to measure wall shear stress in three-dimensional boundary layers. Aeronaut Q 24:87-91

Von Papen T, Steffens H, Ngo HD, Obermeier E (2002) A micro surface fence probe for the application in flow reversal areas. Sens Actuators A Phys 97-98:264-270
Weichert S, Day I (2013) Detailed measurements of spike formation in an axial compressor. J Turbomach 136(5):51006 (TURBO-13-1094)

Young A, Day I, Pullan G (2011) Stall warning by blade passing signature analysis. J Turbomach. https://doi.org/10.1115/GT201 $1-45850$

Publisher's Note Springer Nature remains neutral with regard to jurisdictional claims in published maps and institutional affiliations. 